\title{
Dietary Patterns and CKD Progression
}

\author{
Tanushree Banerjee $^{a} \quad$ Yang Liu $^{b}$ Deidra C. Crews ${ }^{b, c}$ \\ ${ }^{a}$ Division of General Internal Medicine, Department of Medicine, University of California, San Francisco, CA, \\ ${ }^{b}$ Division of Nephrology, Department of Medicine, Johns Hopkins University School of Medicine, and ${ }^{~}$ Welch Center \\ for Prevention, Epidemiology and Clinical Research, Johns Hopkins Medical Institutions, Baltimore, MD, USA
}

\section{Key Words}

Diet · Nutrition · Renal · Kidney · Disparities · Food insecurity

\begin{abstract}
Background: Chronic kidney disease (CKD) patients and their clinicians seek ways to mitigate the risk of CKD progression and its associated complications. Emerging data suggest that dietary modifications may be beneficial adjuvant approaches to reducing the risk of adverse CKD outcomes. Summary: This review focuses on several different dietary patterns, including the Dietary Approaches to Stop Hypertension and Mediterranean diets, and their kidney health benefits. We discuss how healthful dietary patterns are lower in dietary acid load and how improving diet quality may slow the progression of CKD. We also discuss some barriers that may impede socially disadvantaged individuals from following healthful diets. Key Points: Dietary patterns low in dietary acid load might slow the progression of CKD. Current evidence suggests that a reduction in dietary acid load could be beneficial in patients with CKD, but the supremacy of any particular diet is yet to be established. Additional randomized controlled dietary interventions among CKD patients are needed to inform evidence-based recommendations, which can be tailored to an individual's preferences and ability to access healthful foods.
\end{abstract}

(c) 2016 S. Karger AG, Basel

\section{KARGER}

E-Mail karger@karger.com

www.karger.com/bpu

\section{Introduction}

Chronic kidney disease (CKD) is a global public health problem. CKD shares many common risk factors with cardiovascular disease, including some non-modifiable factors, such as age, gender and genetic ancestry. CKD patients and their clinicians seek ways to modify the kidney disease course in order to reduce the risk of developing end-stage renal disease (ESRD), death and other complications of CKD. One such possible avenue is by adopting dietary modifications. Understanding the dietary intake of CKD patients and barriers to its improvement could lead to the formulation of strategies effective at reducing the burden of CKD. In this review, we highlight historical and emerging data on the role of diet in CKD progression with an emphasis on dietary patterns established as beneficial for lowering the risk of cardiovascular diseases.

\section{Micronutrients}

Epidemiologic and clinical evidence has shown linkages between several individual micronutrients and CKD. Dietary interventions focused on reducing the consumption of nutrients such as protein, sodium, and phosphorus have been linked to improved outcomes in $\mathrm{CKD}$, par- 
ticularly in those with moderate to severe disease. According to several studies, dietary protein intake can modulate kidney function, and its role in kidney disease has spawned an ongoing debate in the literature. Based upon 2 meta-analyses, low-protein diets with a dietary protein intake of $0.8 \mathrm{~g} / \mathrm{kg}$ body weight per day reduced the risks of declines in kidney function and/or worsening albuminuria $[1,2]$. However, the optimal level of dietary protein intake to slow the progression of CKD while maintaining adequate nutritional status remains elusive. Further complicating the matter, the source of protein (plant-derived or animal-derived) may have differential effects on kidney function [3]. Protein derived from plant sources, as compared to animal sources, may have less adverse impact on metabolic risk factors in CKD. The metabolic parameters that are affected due to high protein intake are primarily due to high loads of phosphate and nonvolatile acids. Phosphate from plant-based proteins is complexed in the form of phytic acid, which is less digestible in humans and is less bioavailable than animalbased proteins. Additionally, elevated serum phosphorus and serum potassium have been associated with worse outcomes in CKD patients, both in terms of mortality and progression of kidney disease. Thus, control of these two micronutrients has been a long-standing priority in the care of CKD patients.

Yet another emerging public health concern is that of magnesium deficiency. Several lines of evidence suggest that low serum magnesium is associated with incident CKD [4] and kidney function decline [5]. Greater production of inflammatory and pro-atherogenic cytokines in endothelial cells due to low magnesium levels [6] may be a possible pathway contributing to kidney function decline.

Finally, sugar consumption, especially in the form of fructose, has been hypothesized to increase the risk of kidney disease. Investigators have proposed several mechanisms that link sugar consumption with CKD, including increased uric acid, diabetes, obesity and hypertension [7].

\section{Dietary Patterns}

Although intakes of individual micronutrients may play a vital role in CKD outcomes, it can be challenging to quantify them in population-based studies. Further, CKD patients and their clinicians may find it overwhelming to address multiple micronutrients when targeting dietary modifications as a means to slow disease progres- sion. Thus, nephrologists and other clinicians caring for CKD patients might consider assessing the nutritional status of their patients and counseling them on healthful dietary patterns, emphasizing food groups as opposed to micronutrients, in order to influence their risk of CKD progression. Some of the dietary patterns that have been associated with favorable CKD outcomes include the Dietary Approaches to Stop Hypertension (DASH) diet and the Mediterranean diet. They share common features with dietary patterns low in dietary acid load. Table 1 summarizes the findings of some recent studies of the association of healthful dietary patterns with CKD progression and mortality.

\section{DASH Diet}

The DASH dietary pattern, which is endorsed by several national guideline committees, is rich in fruits and vegetables, low fat dairy products, whole grains, fish, poultry, beans, seeds and nuts. It is low in sodium, added sugars, sweets, fats and red meats. The DASH diet is a recognized treatment for hypertension, heart disease, and kidney stones. There are few studies that have examined the association between a DASH dietary pattern and CKD outcomes. In a study of older white women, Lin et al. [8] found that adherence to a DASH-type dietary pattern was associated with lower odds of estimated glomerular filtration rate (eGFR) decline compared with those without a DASH-type dietary pattern. The association remained significant after they accounted for analgesic medication use, lipid-lowering medications and duration of diabetes.

The National Kidney Foundation - Kidney Disease Outcome Quality Initiative (KDOQI) guidelines do not recommend the DASH diet for individuals with 'advanced' CKD (defined as eGFR $<60 \mathrm{ml} / \mathrm{min} / 1.73 \mathrm{~m}^{2}$ ) [9]. This is mainly because the DASH diet contains a higher protein, potassium, and phosphorus content than is recommended for patients with CKD stages 3-4. The optimal daily intake of these minerals for advanced CKD patients, however, is admittedly unknown. Because the DASH trial only enrolled participants with preserved kidney function, the safety and efficacy of the DASH diet for individuals with more advanced kidney disease is largely unknown. In recent work from our group [10], we determined a baseline diet adherence score consistent with DASH diet recommendations, using a 24-hour dietary recall questionnaire in a cohort of 1,110 adults with moderate CKD (eGFR $30-59 \mathrm{ml} / \mathrm{min} / 1.73 \mathrm{~m}^{2}$ ) and hypertension from the National Health and Nutrition Examination Survey III (1988-1994). We related this score to the 
Table 1. Studies reporting effects of different dietary patterns and diet net acid load on progression of CKD and mortality

\begin{tabular}{|c|c|c|c|c|}
\hline Study & Participants & Design & Conclusions & $\begin{array}{l}\text { Overall effect on } \\
\text { kidney function/ } \\
\text { mortality }\end{array}$ \\
\hline $\begin{array}{l}\text { Gutiérrez } \\
\text { et al. [14], } \\
2014\end{array}$ & 3,972 participants with CKD & $\begin{array}{l}\text { Observational } \\
\text { cohort study }\end{array}$ & $\begin{array}{l}\text { Southern dietary pattern rich in } \\
\text { processed and fried foods associated } \\
\text { independently with mortality. Diet rich } \\
\text { in fruits and vegetables appeared to be } \\
\text { protective against mortality }\end{array}$ & $\begin{array}{l}\text { Southern dietary } \\
\text { pattern- } \\
\text { detrimental } \\
\text { Fruits and } \\
\text { vegetables } \\
\text { diet-protective }\end{array}$ \\
\hline $\begin{array}{l}\text { Scialla } \\
\text { et al. [21], } \\
2012\end{array}$ & $\begin{array}{l}632 \text { African American } \\
\text { participants with hypertensive } \\
\text { nephrosclerosis and urine } \\
\text { collections between } 12 \text { and } 36 \\
\text { months }\end{array}$ & Cohort study & $\begin{array}{l}\text { Dietary pattern resulting in higher net } \\
\text { endogenous acid production } \\
\text { associated with a faster rate of CKD } \\
\text { progression }\end{array}$ & $\begin{array}{l}\text { Higher net } \\
\text { endogenous acid } \\
\text { production- } \\
\text { detrimental }\end{array}$ \\
\hline $\begin{array}{l}\text { Kanda } \\
\text { et al. [23], } \\
2014\end{array}$ & $\begin{array}{l}217 \text { CKD patients on low-protein } \\
\text { diet with normal serum } \\
\text { bicarbonate level }\end{array}$ & $\begin{array}{l}\text { Retrospective } \\
\text { cohort study }\end{array}$ & $\begin{array}{l}\text { High net endogenous acid production } \\
\text { independently associated with CKD } \\
\text { progression in elderly CKD patients }\end{array}$ & $\begin{array}{l}\text { Higher net } \\
\text { endogenous acid } \\
\text { production- } \\
\text { detrimental }\end{array}$ \\
\hline
\end{tabular}

development of ESRD ascertained over a median of 14.2 years of follow-up via linkage with the Medicare ESRD Registry. Our results showed a greater risk of ESRD with lower adherence scores - for quintile 1 (lowest adherence): relative hazard (95\% CI) 1.9 (1.4-2.7) when compared with the greatest DASH diet adherence score (quintile 5). The $\mathrm{p}$ for trend across all quintiles was 0.001. Our preliminary findings suggest that the DASH diet may be beneficial for adults with moderate CKD. Randomized trials testing the efficacy and safety of the DASH diet for slowing CKD progression may be warranted.

\section{Mediterranean Diet}

The traditional Mediterranean dietary pattern is characterized by a high intake of vegetables, legumes, fruits, nuts, cereals and olive oil; a moderately high intake of fish; a low-to-moderate intake of dairy products; a low intake of saturated fats, meat and poultry; and a regular but moderate intake of wine during meals. Strong evidence exists demonstrating the beneficial effects of the Mediterranean diet for prevention of several chronic diseases and reduced risk of mortality $[11,12]$. The Mediterranean diet has received attention for ameliorating cardiovascular risk in observational studies and randomized trials. A recent study by Khatri et al. [13] examined the associations of varying levels of adherence to the Mediterranean diet on long-term kidney function in an observational, community-based prospective study and reported that adhering to the diet may significantly reduce the risk of developing CKD. In adjusted models, every 1-point increase in the Mediterranean diet score was associated with decreased odds of incident eGFR $<60 \mathrm{ml} / \mathrm{min} / 1.73 \mathrm{~m}^{2}$ 
(OR (95\% CI) $0.83(0.71-0.96)$ ) and decreased odds of being in the upper quartile of eGFR decline (0.88 (0.79$0.98)$ ).

\section{Other Healthful Dietary Patterns}

Two recently reported studies elucidated the association of other healthful dietary patterns and CKD outcomes. A dietary pattern characterized by fried foods, organ meats, and sweetened beverages, that is, items commonly found in Southern cuisines, has been reported by Gutiérrez et al. [14] to be independently associated with higher risk of mortality among persons with CKD. In contrast, a plant-based dietary pattern high in fruits, vegetables and fish was associated with a lower risk of mortality. Interestingly, in this study, no statistically significant association with risk of ESRD was found for any dietary pattern (which included those labeled as 'convenience', 'sweets', 'Southern', or 'alcohol/salads') [14].

In the Framingham Offspring cohort, Foster et al. [15] evaluated the association between diet quality and rapid kidney function decline. They found that a high diet quality, based on the Dietary Guidelines for Americans score, was associated with both lower odds of incident eGFR $<60 \mathrm{ml} / \mathrm{min} / 1.73 \mathrm{~m}^{2}$ and lower odds of annual eGFR decline $\geq 3 \mathrm{ml} / \mathrm{min} / 1.73 \mathrm{~m}^{2}$.

\section{Dietary Acid Load}

Diet is a major determinant of the acid load that must be excreted by the kidney to maintain acid-base balance. The dietary net acid load has become a key area of focus, and it describes the balance between diet-induced generation of acidity from sulphur-containing amino acids and alkalinity from potassium salts of organic acids [16]. The dietary sulfur content is derived from animal protein intake, which is metabolized to sulfuric acid, whereas potassium salts of organic acids generate bicarbonate. Thus, diets rich in animal protein are acid-producing and increase the dietary net acid load, while diets rich in fruits and vegetables are bicarbonate-generating and decrease the dietary net acid load. Current evidence suggests that a reduction in dietary net acid load could be beneficial in patients with CKD, but the supremacy of any particular diet is yet to be established.

\section{Measurement of Dietary Acid Load}

The effects of diet on acid-base metabolism in humans are sufficiently well established to permit quantitative estimations of net endogenous acid production (NEAP) from knowledge of the types of foods and their amounts consumed. NEAP can be computed from the sum of the production rates of sulfuric acid (resulting from the metabolism of dietary sulfur-containing amino acids) and organic acids (resulting from incomplete combustion of carbohydrate and fat) minus that of bicarbonate (resulting from the combustion of dietary organic acid salts of potassium and magnesium) [17], all of which can be computed from the nutrient composition of individual foods. In typical diets in industrialized countries, the average person excretes acidic urine, indicating the high acid intake in the diet. At higher dietary acid intakes, the body's ability to excrete the total acid load produced seems to be insufficient, and may lead to net acid retention. This is observed with dietary acid loads greater than approximately $1 \mathrm{mEq} / \mathrm{kg}$ body weight [17]. Net acid loads that result from consuming typical American and European diets average around +60 to $+70 \mathrm{mEq} /$ day $[18,19]$, while those of vegetarians and/or fruit and vegetable preferring individuals are usually lower. Figure 1 shows the potential acid load of several commonly consumed foods and beverages.

\section{Dietary Acid Load and CKD Progression}

Multiple small studies by Goraya et al. [20] have found that the provision of fruits and vegetables to reduce dietary acid load among individuals with hypertensive CKD can lead to reductions in markers of kidney injury, including their study of individuals with CKD stage 4, which demonstrated reductions in urinary albumin excretion at 1 year, without increases in serum potassium. Their findings are supported by that of Scialla et al. [21] who noted an association between higher NEAP and faster decline in kidney function among African Americans with hypertensive nephropathy. Additionally, an observational study by our group found an increased risk of CKD progression associated with higher baseline concentrations of dietary acid load [22]. We observed that in the US CKD population, higher levels of dietary acid load were associated with 3 times greater risk of ESRD as compared to those with lower levels (relative hazard (95\% CI) 3.04 (1.58-5.86)). A cohort study conducted in Japan [23] also investigated the association of dietary acid load with CKD progression. In this population with an average age of 70.6 years, the researchers found that high dietary acid load was independently associated with CKD progression (HR (95\% CI) 4.30 (2.10-8.81), for the fourth quartile of dietary acid load compared to the first quartile). Thus, based on this body of evidence, decreasing the dietary acid load may be an effective and safe kidney-protective therapy. 
Fig. 1. Potential renal acid load (PRAL) of selected food items (per $100 \mathrm{~g}$ serving) is adapted from estimates performed by Remer and Manz $[18,19]$. Reprinted, with permission [24].

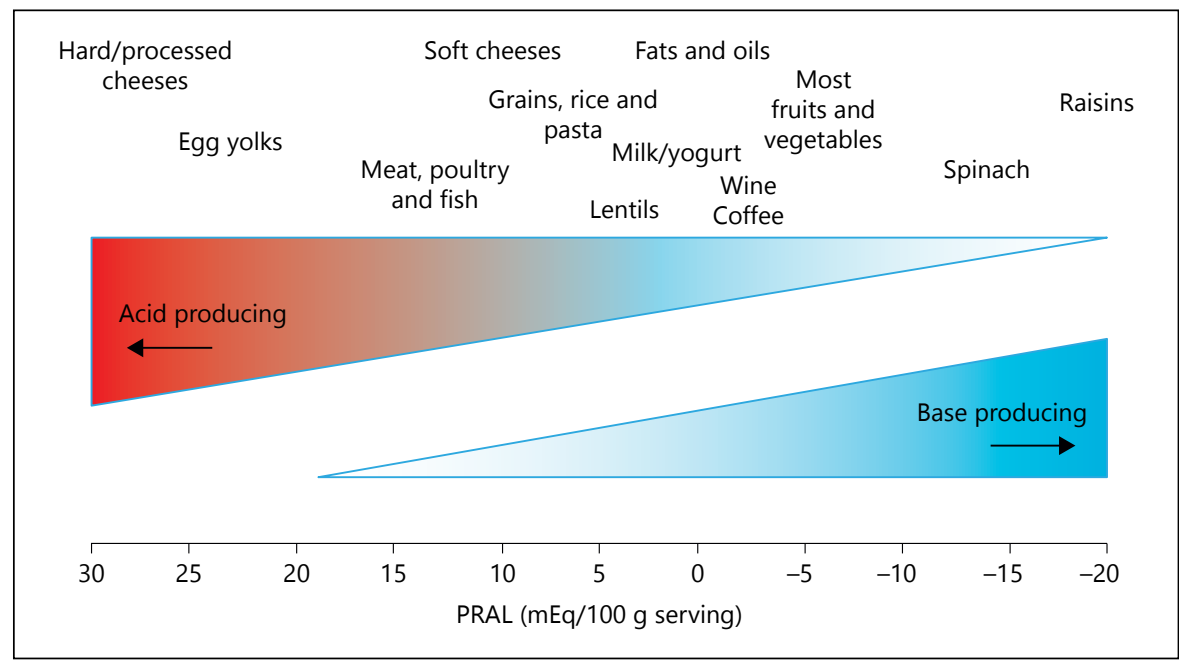

\section{Disparities in Access to Healthful Foods and Dietary Patterns}

CKD patients are frequently encouraged to follow a 'healthy' diet. Such a diet may be particularly difficult to follow if the recommended foods cannot be easily acquired - a situation that individuals living in poverty often face. Food insecurity often accompanies poverty and is associated with several diet-related conditions, including CKD [25] and progression to ESRD [26]. Food-insecure individuals tend to consume more energy-dense foods and relatively inexpensive processed and fast foods enriched with highly absorbable phosphorus additives. Moreover, food-insecure individuals frequently reside in neighborhoods lacking grocery stores most likely to sell healthful foods.

Limited access to nutritious food may be linked to poor dietary quality and ultimately to diet-related diseases. In a study from our group using cross-sectional data [27] from the Baltimore City, Maryland-based Healthy Aging in Neighborhoods of Diversity across the Life Span (HANDLS) study, we studied whether the association of DASH diet adherence and CKD differed between adults living in poverty vs. non-poverty. The lowest DASH adherence tertile (compared with the highest) was associated with greater odds of CKD among the poverty group (OR (95\% CI) 3.15 (1.51-6.56)), but not among the nonpoverty group (0.73 (0.37-1.43)). According to our findings, poor dietary habits were strongly associated with CKD among the urban poor and may represent a target for interventions aimed at reducing disparities in CKD.

When we conducted a qualitative study of socioeconomically disadvantaged African Americans with multi- ple risk factors for $\mathrm{CKD} / \mathrm{CKD}$ progression [28], we found that access to healthful foods was a barrier to dietary modifications that might prevent CKD in this population, as were dietary preferences rooted in familial and cultural practices. Thus, dietary interventions targeting populations at high risk for adverse kidney outcomes should be tailored to address contextual factors that pose challenges to making lifestyle modifications.

\section{Conclusion}

Effective management of CKD should lead to longer survival, decreased morbidity, and improved quality of life. Diet is a daily challenge as well as an educational opportunity that interfaces with all other aspects of effective treatment of CKD. Healthful dietary patterns should be encouraged in patients with $\mathrm{CKD}$, and clinicians managing such patients should seek to understand barriers to dietary adherence that are specific to their culture, geographical region and resources. Plant-based diets and diets rich in fruits and vegetables that are low in dietary net acid load exhibit beneficial metabolic effects in patients with CKD and seem to be safe, although these data were obtained from small translational and observational studies. Future trials are needed to establish the efficacy of dietary interventions alone or in conjunction with other therapies in improving outcomes for CKD patients.

\section{Disclosure Statement}

The authors report no relevant disclosures. 


\section{References}

1 Kasiske BL, Lakatua JD, Ma JZ, Louis TA: A meta-analysis of the effects of dietary protein restriction on the rate of decline in renal function. Am J Kidney Dis 1998;31:954-961.

2 Pedrini MT, Levey AS, Lau J, Chalmers TC, Wang $\mathrm{PH}$ : The effect of dietary protein restriction on the progression of diabetic and nondiabetic renal diseases: a meta-analysis. Ann Intern Med 1996;124:627-632.

3 Scialla JJ, Appel LJ, Wolf M, et al: Plant protein intake is associated with fibroblast growth factor 23 and serum bicarbonate levels in patients with chronic kidney disease: the chronic renal insufficiency cohort study. J Ren Nutr 2012;22:379-388.e1.

4 Tin A, Grams ME, Maruthur NM, et al: Results from the atherosclerosis risk in communities study suggest that low serum magnesium is associated with incident kidney disease. Kidney Int 2015;87:820-827.

5 Van Laecke S, Nagler EV, Verbeke F, Van Biesen W, Vanholder R: Hypomagnesemia and the risk of death and GFR decline in chronic kidney disease. Am J Med 2013;126:825-831.

6 Ferrè S, Baldoli E, Leidi M, Maier JA: Magnesium deficiency promotes a pro-atherogenic phenotype in cultured human endothelial cells via activation of NFkB. Biochim Biophys Acta 2010;1802:952-958.

7 Karalius VP, Shoham DA: Dietary sugar and artificial sweetener intake and chronic kidney disease: a review. Adv Chronic Kidney Dis 2013;20:157-164

8 Lin J, Fung TT, Hu FB, Curhan GC: Association of dietary patterns with albuminuria and kidney function decline in older white women: a subgroup analysis from the nurses' health study. Am J Kidney Dis 2011;57:245-254.

9 Clinical practice guidelines for nutrition in chronic renal failure. K/DOQI, National Kidney Foundation. Am J Kidney Dis 2000;35(6 suppl 2):S1-S140
10 Banerjee T, Crews D, Pavkov M, Burrows NR, Bragg-Gresham J, Saran R, Powe NR: Lower Risk of ESRD Associated with DASH Diet in Adults with Moderate CKD and Hypertension. Paper presented at: American Society of Nephrology, San Diego, Nov 3-8, 2015.

11 Estruch R, Ros E, Salas-Salvadó J, et al: Primary prevention of cardiovascular disease with a Mediterranean diet. N Engl J Med 2013;368:1279-1290.

12 Sofi F, Cesari F, Abbate R, Gensini GF, Casini A: Adherence to Mediterranean diet and health status: meta-analysis. BMJ 2008;337: a1344.

13 Khatri M, Moon YP, Scarmeas N, et al: The association between a Mediterranean-style diet and kidney function in the northern Manhattan study cohort. Clin J Am Soc Nephrol 2014;9:1868-1875.

14 Gutiérrez OM, Muntner P, Rizk DV, et al: Dietary patterns and risk of death and progression to ESRD in individuals with CKD: a cohort study. Am J Kidney Dis 2014;64:204213.

15 Foster MC, Hwang SJ, Massaro JM, Jacques PF, Fox CS, Chu AY: Lifestyle factors and indices of kidney function in the Framingham heart study. Am J Nephrol 2015;41:267-274.

16 Remer T: Influence of diet on acid-base balance. Semin Dial 2000;13:221-226.

17 Lennon EJ, Lemann J Jr, Litzow JR: The effects of diet and stool composition on the net external acid balance of normal subjects. J Clin Invest 1966;45:1601-1607.

18 Remer T, Manz F: Estimation of the renal net acid excretion by adults consuming diets containing variable amounts of protein. Am J Clin Nutr 1994;59:1356-1361.

19 Remer T, Manz F: Paleolithic diet, sweet potato eaters, and potential renal acid load. Am J Clin Nutr 2003;78:802-803; author reply 803-804.
20 Goraya N, Simoni J, Jo CH, Wesson DE: A comparison of treating metabolic acidosis in CKD stage 4 hypertensive kidney disease with fruits and vegetables or sodium bicarbonate. Clin J Am Soc Nephrol 2013;8:371-381.

21 Scialla JJ, Appel LJ, Astor BC, et al: Net endogenous acid production is associated with a faster decline in GFR in African Americans. Kidney Int 2012;82:106-112.

22 Banerjee T, Crews DC, Wesson DE, et al: High dietary acid load predicts ESRD among adults with CKD. J Am Soc Nephrol 2015;26: 1693-1700

23 Kanda E, Ai M, Kuriyama R, Yoshida M, Shiigai T: Dietary acid intake and kidney disease progression in the elderly. Am J Nephrol 2014;39:145-152.

24 Scialla JJ, Anderson CA: Dietary acid load: a novel nutritional target in chronic kidney disease? Adv Chronic Kidney Dis 2013;20:141-149.

25 Crews DC, Kuczmarski MF, Grubbs V, et al: Effect of food insecurity on chronic kidney disease in lower-income Americans. Am J Nephrol 2014;39:27-35.

26 Banerjee T, Crews DC, Wesson D, Dharmarajan $S$, Saran $R$, Burrows $N$, Williams $D$, Powe NR: Association of Food Insecurity and ESRD among a National Cohort of Low-Income Adults with CKD. Paper presented at: American Society of Nephrology, Atlanta, Nov 5-10, 2013.

27 Crews DC, Kuczmarski MF, Miller ER 3rd, Zonderman AB, Evans MK, Powe NR: Dietary habits, poverty, and chronic kidney disease in an urban population. J Ren Nutr 2015; 25:103-110.

28 Johnson AE, Boulware LE, Anderson CA, et al: Perceived barriers and facilitators of using dietary modification for CKD prevention among African Americans of low socioeconomic status: a qualitative study. BMC Nephrol 2014;15:194. 